\title{
Editorial: Multi-Omics Approaches to Study Complex Traits in Domestic Animals
}

\author{
Jianghong $W u^{1 *}$, Xiaolong $\mathrm{Li}^{2}$ and Xiaojiang $\mathrm{Xu}{ }^{3}$ \\ ${ }^{1}$ College of Animal Science and Technology, Inner Mongolia Minzu University, Tongliao, China, ${ }^{2}$ Department of Electronics and \\ Computer Engineering Technology, Indiana State University, Terre Haute, IN, United States, ${ }^{3}$ National Institute of Environmental \\ Health Sciences, National Institutes of Health, Durham, NC, United States
}

Keywords: multi-omics approach, domestic animals, complex traits, gut microbiota, machine learning

Editorial on the Research Topic

Multi-Omics Approaches to Study Complex Traits in Domestic Animals

\section{INTRODUCTION}

Domestic animals are important for human society as they provide the principal protein source in many people's diets. There are notable advantages to dissecting domestic animals' genetic basis for complex traits, as we have access to more details of phenotype records than other animals. The population of domestic animals has a rich collection and mutation of affected phenotypic traits (Andersson and Georges, 2004). Research on domestic animals has entered a

OPEN ACCESS

Edited and reviewed by: Rongling $\mathrm{Wu}$,

The Pennsylvania State University (PSU), United States

*Correspondence: Jianghong Wu wujianghonglong@126.com

Specialty section: This article was submitted to Integrative Genetics and Genomics, a section of the journal

Frontiers in Systems Biology

Received: 06 September 2021 Accepted: 27 September 2021

Published: 15 October 2021

Citation:

Wu J, Li X and Xu X (2021) Editorial: Multi-Omics Approaches to Study Complex Traits in Domestic Animals. Front. Syst. Biol. 1:771644. doi: 10.3389/fsysb.2021.771644 post-genomic era, which is difficult for scientists to study and understand because complex traits are influenced by many genetic and environmental factors. After a decade of genome-wide association studies, fundamental questions on domestic animal genetics, such as the extent of pleiotropy across the genome and variation in genetic architecture across complex traits, are still unanswered.

Multi-omics is a powerful technology to understand the interaction between genotype, environment, and life in a concerted way and illustrates these complex traits and diseases. The omics field has been driven largely by technological advances that have made cost-efficient, highthroughput analysis of biological molecules possible. Such omics data are derived from diverse biological sources and include metagenomics, genomics, transcriptomics, epigenomics, proteomics, metabolomics, and so on. The integration of omics data provides a better understanding of how a genotype influences a complex phenotype, as well as the molecular mediators at the multiscale that regulate the underlying pathway mechanisms of complex traits in domestic animals. The Research Topic on "Multi-omics Approaches to Study Complex Traits in Domestic Animals" in Frontiers in Genetics includes a series of four articles that discuss recent advances regarding the underlying regulation mechanisms of complex traits in domestic animals and highlight challenges and outstanding questions that need to be addressed in future research.

\section{MULTI-OMICS WITHIN DOMESTIC ANIMALS}

After a decade of genome-wide association studies, a large number of genetic variants associated with specific traits have been discovered. However, the difficulty of detecting GWAS results for 
complex traits may be influenced by the pleiotropic nature of these traits. Integrating other types of data such as intermediate molecular phenotypes to identify associations could overcome these limitations. Liu et al. performed a genome-wide association study and RNA sequencing analyses to detect candidate genes for the feather rate in Shouguang chicken. Two overlapping genes (SPEF2 and PRLR) between the results of GWAS and differential expression analysis were identified, which provide possible candidate genes for the formation of the chicken feathering phenotype. Wu et al. investigated clinical ketosis-associated metabolomic and proteomic changes using statistical analyses. Ketosis altered a wide range of metabolic pathways, such as metabolism, metabolism of proteins, gene expression and post-translational protein modification, vitamin metabolism, signaling, and disease-related pathways. Integration of Multiomics was utilized in the future study to 1) investigate the extent of pleiotropy at complex traits, and 2) to characterize the hub molecular (gene, SNP, and gene-set levels gene or metabolite) trait-associated variants and crosstalk among different regulation levels of biological functionality of complex traits.

\section{MULTI-OMICS BETWEEN DOMESTIC ANIMALS AND GUT MICROBIOTA}

The review by Patil et al. (2020) discusses the interactions between host and gut microbiota in domestic animals. The microbiome plays a critical role in maintaining metabolic homeostasis, neurological and immunological functions, including protection from pathogens and digestion of food materials. Seika and Theresa also review microbiome genomic data across domestic species and highlight the common occurrence of gut microbiome dysbiosis during idiopathic intestinal inflammation in multiple species. Domestic animals represent important resources for understanding the shared mechanisms underlying complex natural diseases that arise due to both genetic and environmental factors. The gut microbiota also plays a critical role in the digestion of food materials, especially for a ruminant. Wu et al. used Multi-omics techniques to analyze the nutritional value of different lamb feed from the microbiome, transcriptome, metabolome, and fatty acid profiles. They found that there was potential cross-talk between the four omics data layers, which helps to further understanding of the mechanism by which feed affects the meat quality of lamb (Wu et al., 2020). While deeper analyses of different levels of omics data in a host, coupled with microbiome molecular analyses in the digestion track are

\section{REFERENCES}

Andersson, L., and Georges, M. (2004). Domestic-animal Genomics: Deciphering the Genetics of Complex Traits. Nat. Rev. Genet. 5, 202-212. doi:10.1038/nrg1294 needed, comparative studies across domestic species can reveal shared microbial alterations and regulatory mechanisms that will improve understanding of complex traits in domestic animals.

\section{FUTURE PERSPECTIVES}

Multi-omics approaches derive a holistic understanding of the regulation mechanism under complex traits in domestic animals with its share of challenges. On the one hand, Multiomics data are generated from different platforms with various data formats. How to integrate the different data layers of Multi-omics as a system should be considered. It is a challenge to combine machine learning in the data preprocessing of Multi-omics and to improve accuracy and inject biological information. On the other hand, the genetic dissection of complex traits, such as meat quality and residual feed intake, are challenging because of the small effect of each locus and the interaction of environmental factors that can affect the phenotype. A systematic analysis of Multi-omics data and an experimental design are critical components of the genetic dissection of complex traits and new systems biology tools and pipelines for Multi-omics need to be developed to address these challenges in the next decade.

\section{AUTHOR CONTRIBUTIONS}

$\mathrm{XL}$ and XX polished this manuscript. JW compiled the contributions from all authors. All authors contributed to the article and approved the final version of the manuscript.

\section{FUNDING}

This work was supported by the National Natural Science Foundation of China (31560623), the Doctoral Scientific Research Foundation of Inner Mongolia University for Nationalities (BS527), and the Key Science-Technology Project of Inner Mongolia (2021GG0008).

\section{ACKNOWLEDGMENTS}

We would like to thank all authors for their contributions to the Research Topic and the reviewers for their time and effort. We also greatly appreciate the Chief Editor and Editorial Office of Frontiers in Genetics for their support during the process of processing the manuscript.

Patil, Y., Gooneratne, R., and Ju, X.-H. (2020). Interactions between Host and Gut Microbiota in Domestic Pigs: a Review. Gut Microbes 11 (3), 310-334. doi:10.1080/19490976.2019.1690363

Wu, J., Yang, D., Gong, H., Qi, Y., Sun, H., Liu, Y., et al. (2020). Multiple Omics Analysis Reveals that High Fiber Diets Promote Gluconeogenesis and 
Inhibit Glycolysis in Muscle. BMC Genomics 21, 660. doi:10.1186/s12864020-07048-1

Conflict of Interest: The authors declare that the research was conducted in the absence of any commercial or financial relationships that could be construed as a potential conflict of interest.

Publisher's Note: All claims expressed in this article are solely those of the authors and do not necessarily represent those of their affiliated organizations, or those of the publisher, the editors and the reviewers. Any product that may be evaluated in this article, or claim that may be made by its manufacturer, is not guaranteed or endorsed by the publisher.

Copyright $\odot 2021 \mathrm{Wu}, \mathrm{Li}$ and $\mathrm{Xu}$. This is an open-access article distributed under the terms of the Creative Commons Attribution License (CC BY). The use, distribution or reproduction in other forums is permitted, provided the original author(s) and the copyright owner(s) are credited and that the original publication in this journal is cited, in accordance with accepted academic practice. No use, distribution or reproduction is permitted which does not comply with these terms. 